\title{
MATHEMATICAL MODELING OF SHORT PULSED LASER IRRADIATION IN THE CORNEA: A DUAL PHASE LAG MODEL
}

\author{
Ram Autar, Anuj Kumar \\ Department of Mathematics, Harcourt Butler Technical University \\ Kanpur 208002, India \\ rautar62@gmail.com,kumaranuj979588@gmail.com
}

Received: 1 March 2021; Accepted: 12 August 2021

\begin{abstract}
A simple mathematical model for the temperature evolution in the cornea exposed to short-pulsed Ho: YAG laser under Laser Thermo Keratoplasty (LTK) treatment is developed by incorporating both the heat flux phase-lag and temperature gradient phase-lag in Fourier's heat transfer model. An analytical solution to the mathematical model is obtained using the Laplace transformation technique. The computational results for the temperature profile and the temperature variation with time are presented through the graphs. The effect of some typical parameters: the heat flux phase-lag and the temperature gradient phase-lag on the temperature distribution and temperature variations are illustrated and discussed.
\end{abstract}

MSC 2010: 92B05, 80A20, 78A60, 35L20

Keywords: temperature, laser, cornea, refractive error, LTK, Laplace transformation technique, dual phase lag equation

\section{Introduction}

Nowadays, the refractive error of the eye due to a pathological state of hyperopia in which light is focused too far behind the retina also is treated with a short-pulsed laser that recontours the corneal curvature, making it steeper as required to improve the patient's refractive error. In this refractive procedure, holmium: yttrium aluminium garnet laser thermo keratoplasty (Ho: YAG LTK) is a frequently used thermal procedure, and the heat is used to reshape the surface of the cornea through the shrinkage of collagen within the stroma. Ho: YAG is a family of solid state lasers that use the doping element holmium in a laser crystal YAG and that emit energy at approximately 2.1 microns. It is a versatile laser with multiple soft tissue applications including tissue incision and vapourization, the holmium produces immediate tissue vapourization while minimizing deep thermal damage to surrounding tissues. Ho: YAG LTK is a quick procedure with little risk of infection and discomfort. It is directed towards the creation of safe localised heating in the central stroma. 
One of the most important challenges in thermal therapy is to deliver the appropriate amount of heat to the targeted part of corneal tissue. If the temperature becomes too high, or if they are kept elevated for a long time, then there may be serious side effects. Hence, it is important to find out the temperature field in the cornea for having better control of the process and for the safety of corneal endothelium. Moreover, determining the temperature distribution in such a condition is important for the treatment planned and for designing new clinical systems. With the development of high performance computing systems, the mathematical models for the temperature evolution in biological tissues subjected to thermal therapies such as laser irradiation and the analysis of simulation results are growing in sophistication and are thereby becoming better to quantify and understand the thermal response of the tissues.

In recent years, several bioheat transfer models for temperature rise in biological tissues have been developed and simulated. Hobiny and Abbas [1], Alzahrani and Abbas [2] and Abbas, Hobiny and Alzaharani [3] have used the Laplace transformation technique to obtain analytical solutions to their hyperbolic bioheat transfer models under different conditions, while Hobiny and Abbas [4] have used the finite element approach to solve their bioheat model. Ghanami and Abbas [5], Hobiny and Abbas [6] and Hobiny et al. [7] developed fractional bioheat transfer models to determine the temperature and the thermal damage in the tissue and obtained analytical solutions to the models using the Laplace transform. In 2020, Hobiny and Abbas [8] used the finite element approach to find a numerical solution to their nonlinear dual phase lag bioheat model for the temperature changes and evaluated the thermal damage using the Arrhenius equation. In these works, it has been concluded that mathematical models are efficient tools to evaluate the bioheat transfer in living tissues.

Numerous experimental [9] and theoretical [10] investigations have been carried out to study the thermomechanical behaviour of the human cornea under the LTK treatment to improve the predictability and the repeatability of the reliable clinical results and minimize/reduce irreversible thermal damage to the cornea. In 1979, Mainster [11] developed a mathematical model for the temperature changes in the cornea during the LTK surgery. He assumed the cornea as a semi-infinite region and one-dimensional heat transfer in the tissue region. Rahbar and Shokooh-Saremi [12] presented a mathematical analysis of the thermal effects of the excimer laser on the anterior side of the human eye. They considered the linear effect of an absorption coefficient and the actual dimension of the human eye. Brinkmann et al. [13] investigated the corneal temperature under LTK from both the experimental and numerical perspectives. Gokul et al. $[14,15]$, used finite element models to investigate the temperature distribution of the cornea in different laser refractive surgeries and the thermal effects of eyelid closure and opening in the human eye. The characteristics of optical and thermal parameters of laser action are investigated.

Manns et al. [16, 17] developed mathematical models for the temperature distribution in the cornea under LTK surgery assuming the cornea as a single-layered tissue slab in the two-dimensional coordinate system. Analytical solutions to the models were obtained. The thermal load during ablation in high-speed laser corneal refractive surgery is evaluated with an excimer laser by Ortueta et al. [18]. They found that the peak temperature of the corneal surface never exceeded $35^{\circ} \mathrm{C}$ in any case. Shibib [19] 
used the finite element method to predict, numerically, the temperature distribution in the eye when subjected to a laser beam. The effects of laser parameters on the temperature distribution and the consequential thermal damage were studied.

Gheitaghy et al. [20] developed the hyperbolic and parabolic models for the heat transfer in a non-perfused homogeneous transparent cornea under ultrashort pulsed laser irradiation in the LTK treatment. The models were solved by exploiting the mathematical analogy between thermal and electrical systems. They concluded that the hyperbolic wave model predicted a higher temperature than that predicted by the classical heat diffusion model. Narasimhan and Sadasivam [21] developed a dual phase lag model for heat conduction in the eye subjected to retinal laser irradiation. The computational results for the temperature distribution and thermal damage distribution are obtained and the effect of some model parameters on the temperature are illustrated and discussed.

The corneal tissue consists of a complicating and non-homogeneous structure. Not only is a time delay between the high heat flux and the temperature gradient but also a time delay between the temperature gradient and the energy transport. The dual phase lag (DPL) model of heat conduction which takes into consideration both the relaxation times of the heat flux vector and the temperature gradient should be combined with the Pennes' bioheat equation to formulate a mathematical model for the temperature evolution in the corneal tissue under LTK surgery. The present work is concerned with the development of a mathematical model for the corneal temperature during LTK surgery by taking into account both the heat flux phase-lag and the temperature gradient phase-lag in the model. The non-linear dual phase-lag model is solved using the Laplace transform method.

\section{Physical model and mathematical formulation}

The cornea is modeled as a finite domain exposed to a short-laser heat pulse at the front surface (left boundary of Fig. 1) as in the Ho: YAG LTK surgery. The energy of the laser beam which is incident at the centre of the cornea is assumed to be absorbed in the stroma. One-dimensional heat transfer is assumed.

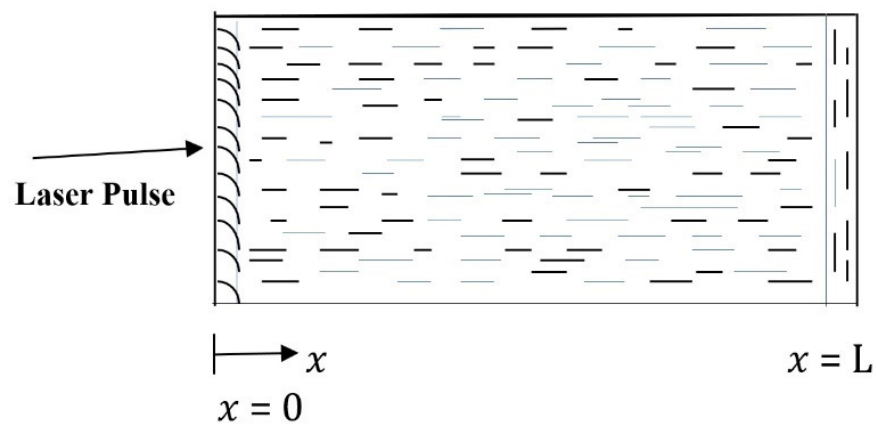

Fig. 1. Schematic diagram of the cornea modeled as a finite domain exposed to Ho: YAG short-pulsed laser 
The elimination of heat flux term in Pennes' model [22]:

$$
\rho c \frac{\partial T(x, t)}{\partial t}=-\nabla \cdot q+\rho_{b} c_{b} \omega_{b}\left(T_{b}-T\right)+q_{m b}+Q(x, t)
$$

for the heat transfer in biological tissues by adding the heat flux phase-lag $\tau_{q}$ to the time variable $t$ in the heat flux term and the temperature gradient phase-lag $\tau_{T}$ to the variable $t$ in the temperature gradient in Fourier's law of heat conduction:

$$
q(x, t)=-k \frac{\partial T(x, t)}{\partial x}
$$

and taking the first order approximation of Taylor's series expansion with respect to the time of these terms yields the following partial differential equation which governs the temperature evolution in a nonperfused avascular homogeneous cornea irradiated by the short-pulsed Ho: YAG laser in the Laser Thermo Keratoplasty (LTK) surgery.

$\tau_{q} \frac{\partial^{2} T(x, t)}{\partial t^{2}}+\frac{\partial T(x, t)}{\partial t}=\alpha \frac{\partial^{2} T(x, t)}{\partial x^{2}}+\alpha \tau_{T} \frac{\partial^{3} T(x, t)}{\partial x^{2} \partial t}+\frac{1}{\rho c}\left[Q+\tau_{q} \frac{\partial Q(x, t)}{\partial t}\right]$

where: $\alpha=\frac{k}{\rho c}, T\left[{ }^{\circ} \mathrm{C}\right]$ is the corneal temperature, $\alpha\left[\mathrm{cm}^{2} \mathrm{~s}^{-1}\right]-$ the thermal diffusivity of the corneal tissue, $\rho\left[\mathrm{kg} \mathrm{m}^{-3}\right]$ - the density of corneal tissue, $c\left[\mathrm{~J} \mathrm{~kg}^{-1} \mathrm{~K}^{-1}\right]-$ the specific heat of corneal tissue, $k\left[\mathrm{~W} \mathrm{~m}^{-1}{ }^{\circ} \mathrm{C}^{-1}\right]$ - the thermal conductivity of corneal tissue, $Q(x, t)\left[\mathrm{W} \mathrm{m}^{-3}\right]$ - the heat generation due to the laser and $x, t$ are the space co-ordinate and time, respectively. The laser heat source $Q(x, t)$ is described as follows.

$$
Q(x, t)=I(t)(1-\mathrm{R}) \mu e^{-\mu x}
$$

where: $\mathrm{R}$ is Fresnel surface reflectance, $\mu\left[\mathrm{m}^{-1}\right]$ - the absorption coefficient of the corneal tissue and $I(t)\left[\mathrm{W} \mathrm{m}^{-2}\right]$ - the laser intensity. The laser heat source is assumed to be Gaussian and has been separated in variables: time and space.

The physically realistic and mathematically consistent initial and boundary conditions are prescribed as follows:

$$
\begin{aligned}
T(x, t)=T_{0}, \frac{\partial T(x, t)}{\partial t}=0, & \text { at } t=0 . \\
-k \frac{\partial T(x, t)}{\partial x}=h\left(T-T_{0}\right)+\sigma \epsilon\left(T^{4}-T_{0}{ }^{4}\right)+\mathrm{E}, & \text { at } x=0 \\
\frac{\partial T(x, t)}{\partial x}=0, & \text { at } x=L
\end{aligned}
$$


where: $T_{0}\left[{ }^{\circ} \mathrm{C}\right]$ is the initial corneal temperature, $\mathrm{E}\left[\mathrm{W} \mathrm{m}^{-2}\right]-$ the evaporative heat loss, $\sigma\left[\mathrm{W} \mathrm{m}^{-2} \mathrm{~K}^{-4}\right]$ - the Stefan Boltzmann constant, $h\left[\mathrm{~W} \mathrm{~m}^{-2} \mathrm{~K}^{-1}\right]-$ the convection coefficient and $\varepsilon$ the emissivity of the cornea.

The boundary condition (4) represents that the heat loss at the anterior surface of the cornea occurs due to convection, emission and evaporation. The boundary condition (5) states that the posterior surface of the cornea is thermally insulated.

The governing equation with initial and boundary conditions is normalised using the following scheme:

$$
\mathrm{X}=\frac{\omega x}{2 \alpha}, \quad \tau=\frac{t}{2 \tau_{q}}, \quad \psi=\frac{Q \tau_{q}}{\rho c T_{0}}, \quad \theta=\frac{T}{T_{0}},
$$

and is written in the dimensionless form:

$$
\frac{\partial^{2} \theta}{\partial \tau^{2}}+2 \frac{\partial \theta}{\partial \tau}=\frac{\partial^{2} \theta}{\partial \mathrm{X}^{2}}+\frac{\tau_{T}}{2 \tau_{q}} \frac{\partial^{3} \theta}{\partial \mathrm{X}^{2} \partial \tau}+4 \psi_{0} \eta(\tau) e^{-\beta \mathrm{X}}+2 \frac{\partial \psi_{0} \eta(\tau) e^{-\beta \mathrm{X}}}{\partial \tau}
$$

where: $\Psi(\mathrm{X}, \tau)=\psi_{0} \eta(\tau) e^{-\beta \mathrm{X}}, \psi_{0}=\frac{\beta I_{r}(1-R \mathrm{R})}{2 \omega \rho c T_{0}}, \beta=2 \omega \tau_{q} \mu$, and $\eta(\tau)$ is the dimensionless rate of energy absorbed in the medium, the laser heat source term is expressed in terms of arbitrary reference laser intensity $I_{r}$ as

$$
\begin{aligned}
& I(\tau)=I_{r} \eta(\tau) \\
& \theta(\mathrm{X}, \tau)=1, \quad \frac{\partial \theta(\mathrm{X}, \tau)}{\partial \tau}=0, \quad \text { at } \tau=0 \\
& -B \frac{\partial \theta}{\partial \mathrm{X}}=F(\theta-1)+H\left(\tilde{\theta}^{2}+1\right)(\tilde{\theta}+1)(\theta-1)+G, \quad \text { at } \mathrm{X}=0 . \\
& \frac{\partial \theta}{\partial \mathrm{X}}=0, \quad \text { at } \mathrm{X}=\frac{\omega \mathrm{L}}{2 \alpha} \text {. }
\end{aligned}
$$

where: $F=\frac{h T_{0}}{I_{r}}, H=\frac{\sigma \epsilon T_{0}{ }^{4}}{I_{r}}, G=\frac{E \mathrm{E}}{I_{r}}, B=\frac{\omega \rho c T_{0}}{2 I_{r}}$.

\section{Solution to the mathematical model}

The partial differential equation (6) subject to the initial conditions $(7 \mathrm{a}, \mathrm{b})$ and boundary conditions $(8 \mathrm{a}, \mathrm{b})$ is solved using the Laplace transform technique, and the analytical expression for the temperature is obtained as follows:

$$
\begin{aligned}
\theta(\mathrm{X}, \tau)= & \psi_{0} \beta e^{-\beta \mathrm{L}^{\prime}} h\left(\mathrm{~L}^{\prime}-\mathrm{X}, \tau\right)+\psi_{0} e^{-\beta \mathrm{X}} f_{H}+\psi_{0} \beta e^{-\beta \mathrm{L}^{\prime}} \sum_{n=0}^{\infty} h\left((1-2 n) \mathrm{L}^{\prime}-\right. \\
& \mathrm{X}, \tau)-\frac{G}{B} \sum_{n=0}^{\infty} h_{2}\left(-\left(2 n \mathrm{~L}^{\prime}+\mathrm{X}\right), \tau\right)+\psi_{0} \frac{(B \beta-F-R)}{B} \sum_{n=0}^{\infty} h\left(-\left(2 n \mathrm{~L}^{\prime}+\right.\right. \\
& \mathrm{X}), \tau)+\psi_{0} \beta e^{-\beta \mathrm{L}^{\prime}} \sum_{n=0}^{\infty} h\left(-\left(2 n \mathrm{~L}^{\prime}+\mathrm{L}^{\prime}-\mathrm{X}\right), \tau\right)+ \\
& \psi_{0} \frac{(B \beta-F-R)}{B} \sum_{n=0}^{\infty} h\left(-\left(2 n \mathrm{~L}^{\prime}+2 \mathrm{~L}^{\prime}-\mathrm{X}\right), \tau\right)-\frac{G}{B} \sum_{n=0}^{\infty} h_{1}\left(-\left(2 n \mathrm{~L}^{\prime}+\right.\right. \\
& \left.\left.2 \mathrm{~L}^{\prime}-\mathrm{X}\right), \tau\right)+1
\end{aligned}
$$


where: $f_{H}(\tau)=\frac{\gamma_{p e}^{2} \gamma_{m} \tau-\gamma_{m}^{2} e^{-\gamma_{p} \tau}-4 \gamma}{\gamma \gamma_{p} \gamma_{m}}, \gamma=\frac{\left[\left(2-\beta^{2} D\right)^{2}+4 \beta^{2}\right]^{\frac{1}{2}}}{2}, \mathrm{~L}=\frac{\omega L}{2 \alpha}$,

$$
\begin{array}{rlrl}
\gamma_{p} & =\gamma-\frac{\left[2-\beta^{2} D\right]}{2}, \quad \gamma_{m}=\gamma+\frac{\left[2-\beta^{2} D\right]}{2}, & \\
h(p, \tau) & =0, & p \geq \tau \geq 0 \\
& =\int_{p}^{\tau} e^{-u} I_{0}\left[\left(u^{2}-p^{2}\right)\right]^{\frac{1}{2}} f_{H}(\tau-u) d u, & \tau>p \\
h_{i}(p, \tau) & =0, & p \geq \tau \geq 0 \\
& =\int_{0}^{\tau} e^{-(\tau-u)} I_{0}\left[(\tau-u)^{2}-p^{2}\right]^{\frac{1}{2}} d u, & & \tau>p \\
\mathrm{i}=1,2
\end{array}
$$

\section{Results and discussion}

The computational results of the DPL model for the temperature profile and temperature variation with time in the corneal tissue irradiated by Ho: YAG laser under LTK treatment are obtained using the values of typical parameters given in Table 1 and presented through the graphs.

Table 1. Parameters used in computations

\begin{tabular}{|l|c|c|c|}
\hline \multicolumn{1}{|c|}{ Parameter } & Symbol & Magnitude & Dimension \\
\hline Corneal density & $\rho$ & 1062 & $\mathrm{~kg} \mathrm{~m}^{-3}$ \\
\hline Corneal thermal conductivity & $k$ & 0.556 & $\mathrm{~W} \mathrm{~m}^{-1}{ }^{\circ} \mathrm{C}^{-1}$ \\
\hline Fresnel surface reflectance & $R$ & $2.4 \%$ & - \\
\hline Absorption coefficient & $\mu$ & 2000 & $\mathrm{~m}^{-1}$ \\
\hline Thermal diffusivity & $\alpha$ & 0.001452 & $\mathrm{~cm}^{2} \mathrm{~s}^{-1}$ \\
\hline Emissivity of cornea & $\varepsilon$ & 0.975 & - \\
\hline Stefan Boltzmann constant & $\sigma$ & $5.67 \times 10^{-8}$ & $\mathrm{~W} \mathrm{~m}^{-2} \mathrm{~K}^{-4}$ \\
\hline Wavelength & $\lambda$ & $1.85-2.1$ & $\mu \mathrm{m}^{-1}$ \\
\hline Specific heat & $c$ & 3830 & $\mathrm{~J} \mathrm{Kg}^{-1} \mathrm{~K}^{-1}$ \\
\hline Initial temperature & $T_{0}$ & 35 & ${ }^{\circ} \mathrm{C}$ \\
\hline Evaporative heat loss & $E$ & 40 & $\mathrm{~W} \mathrm{~m}^{-2}$ \\
\hline Phase-lag in heat flux & $\tau_{q}$ & 1 & $\mathrm{~s}$ \\
\hline Phase-lag in temperature gradient & $\tau_{T}$ & 0.043 & $\mathrm{~s}$ \\
\hline Thickness of cornea & $L$ & 0.55 & $\mu \mathrm{m}^{-2}$ \\
\hline Reference laser intensity & $I_{r}$ & $2 \times 10^{4}$ & $\mathrm{~W} \mathrm{~m}^{-2}$ \\
\hline Convection coefficient & $h$ & 20 & $\mathrm{~W} \mathrm{~m}^{-2} \mathrm{~K}^{-1}$ \\
\hline Pulse duration & $t_{i}$ & 200 & $\mu \mathrm{s}$ \\
\hline
\end{tabular}


In the actual treatment of LTK, the cornea heating is done at eight different spots which are arranged in an annular pattern about the centre of the corneal surface. The curves in Figure 2 show the distribution of dimensionless temperature along with the dimensionless corneal depth following each laser pulse of the treatment. It is observed that the temperature in the epithelium layer of the cornea predicted by the DPL model is found to lie between 85 and $98^{\circ} \mathrm{C}$ whereas the stromal temperature ranges from 68 to $94^{\circ} \mathrm{C}$. The temperature in the endothelial layer is predicted to lie between 60 and $74^{\circ} \mathrm{C}$. The temperature in various layers of the corneal tissue under LTK treatment increases with the number of pulses.

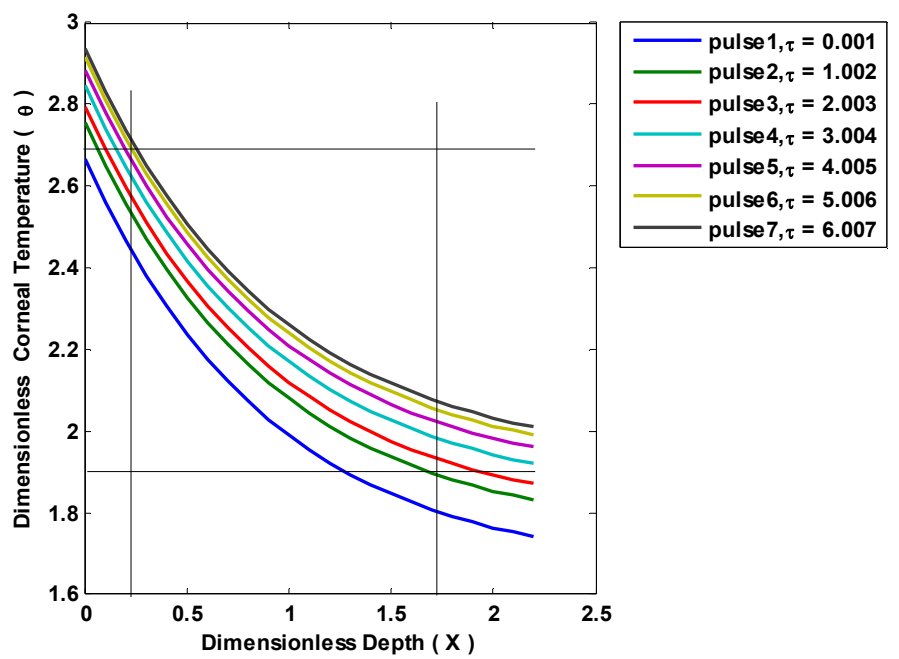

Fig. 2. Dimensionless temperature profiles after each laser pulse, predicted by DPL model

The effect of the phase-lag in heat flux on the corneal temperature distribution at different times is illustrated in Figures 3a-c. As observed, the corneal temperature rises with an increase in the phase-lag in heat flux. The temperature decreases with time, but the effect of relaxation time is observed almost the same.

The temperature variations with time at different interfaces of the cornea are depicted in Figure 4 after the heating phase. The temperature, at the epithelium-stroma interface, decreases with time significantly. However, the temperature at higher depths, i.e.in middle of the stroma and stroma-endothelium interface, increases slightly linearly during the cooling period under consideration.

The effect of the phase-lag in the heat flux on the normalized temperature variation with time is portrayed in Figure 5. It is observed from the curves in the Figure that an increase in the phase-lag in the heat flux increases the temperature.

The effect of the phase-lag in the temperature gradient on the corneal temperature variation with time predicted by the DPL model is shown in Figure 6. It is observed that an increase in the phase-lag temperature gradient reduces the temperature. Also, the rate of temperature decrease with time slows with an increase in the parameter, $\tau_{T}$. 
a)

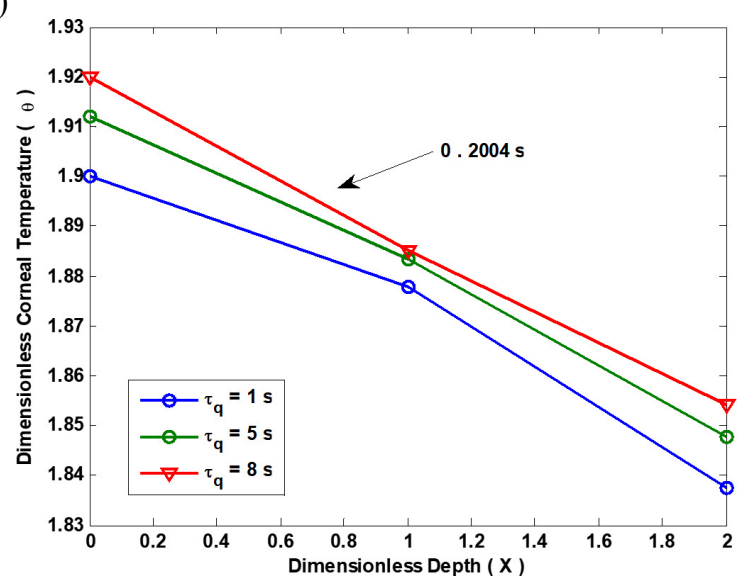

b)

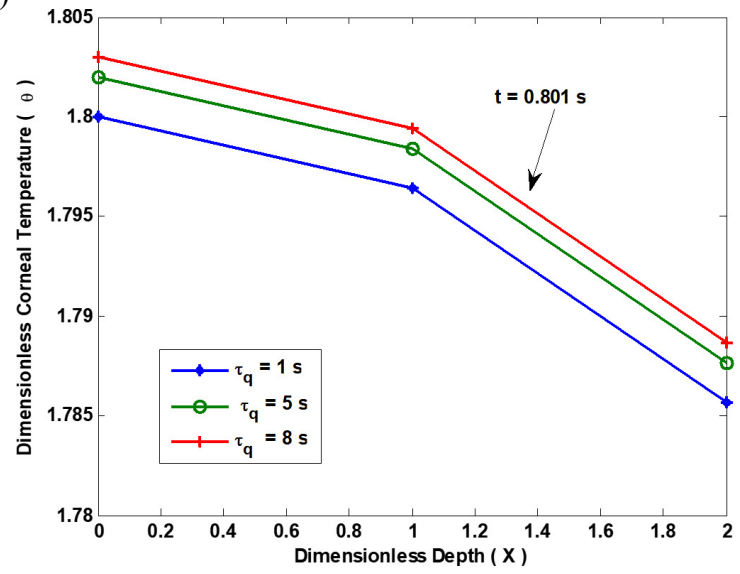

c)

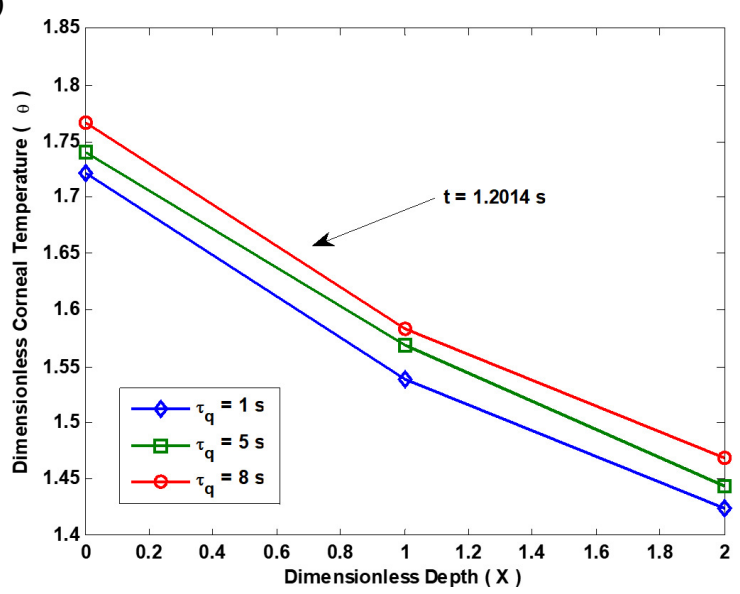

Fig. 3. The sensitivity of the corneal temperature distribution to the phase-lag in heat flux at: a) $t=0.2004 \mathrm{~s}, \mathrm{~b}) t=0.801 \mathrm{~s}, \mathrm{c}) t=1.2014 \mathrm{~s}$ 


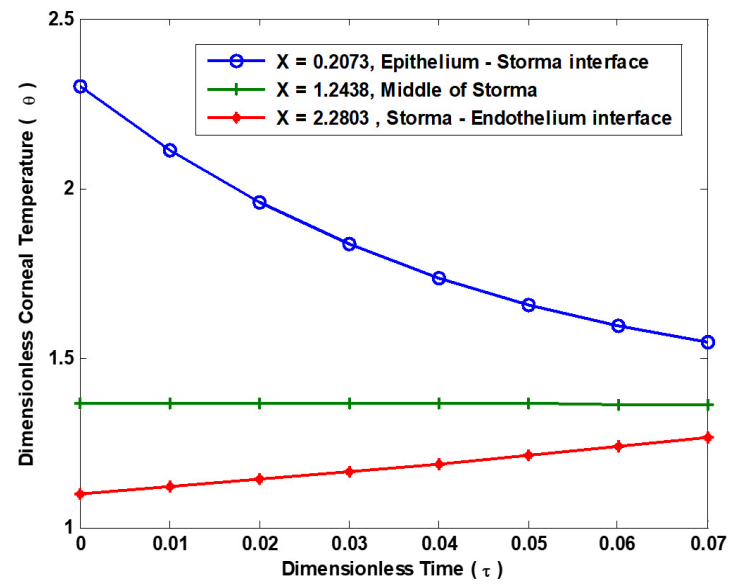

Fig. 4. The temperature variation with time at different interfaces of the corneal tissue during pulsed Ho: YAG laser irradiation

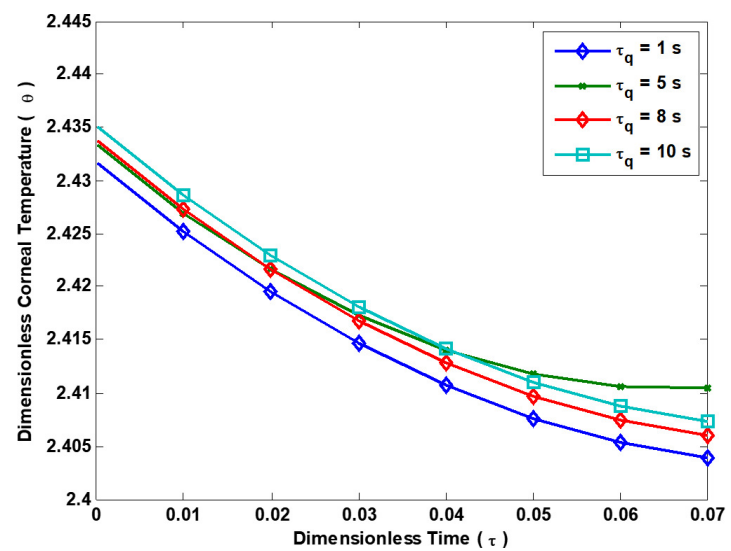

Fig. 5. The effect of the phase-lag in heat flux on the temperature variation with time

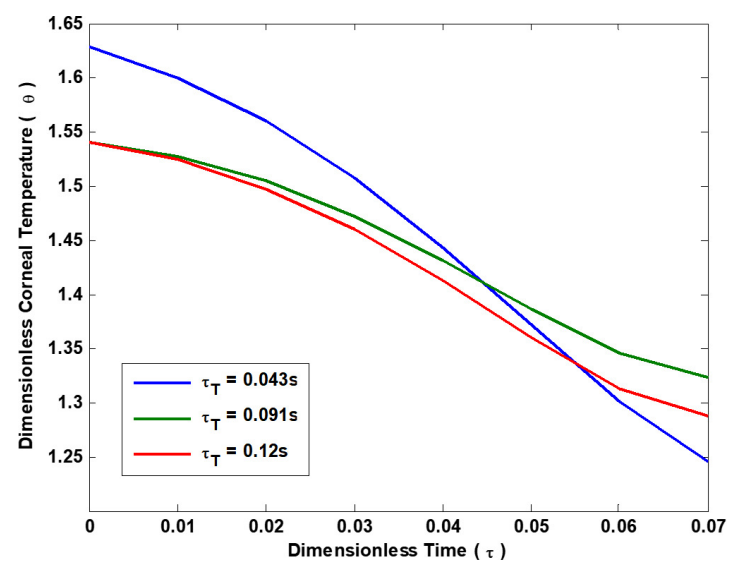

Fig. 6. Effect of the phase-lag in the temperature gradient on the temperature variation time on the anterior surface of the corneal tissue 
A comparison of the corneal temperatures variation with time predicted by the parabolic model, the hyperbolic model and the DPL model is illustrated in Figure 7. The temperature response predicted by Fourier's Model (Parabolic model) decreases with time rapidly whereas the temperature decrease with time predicted by both the hyperbolic model and the DPL model slows down. Most of the time, the temperature predicted by the DPL model is higher than that predicted by the hyperbolic model or parabolic model.

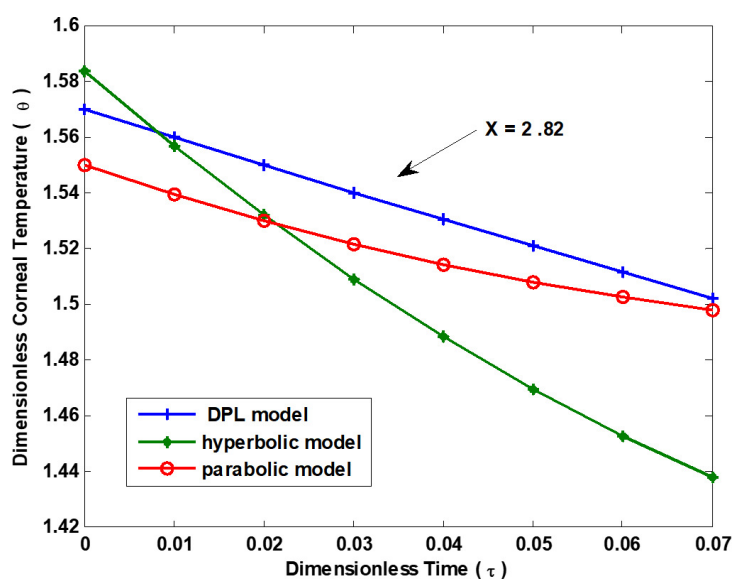

Fig. 7. Comparison of the dimensionless temperature variations with time predicted by the Fourier's model, hyperbolic model and DPL model at $x=0.5 \mathrm{~mm}$ depth of the corneal tissue during pulsed Ho: YAG laser irradiation

\section{Conclusion}

The dual phase lag heat conduction model is used to describe the temperature changes in the human cornea exposed to short-pulsed Ho: YAG laser heating under LTK surgery and to investigate the effects of the phase-lags in the heat-flux and the temperature gradient on the corneal temperature distribution and temperature variation with time in this study. As observed from the computational results, the temperature in the epithelium layer lies between 85 and $98^{\circ} \mathrm{C}$, the stromal temperature lies between 68 and $94^{\circ} \mathrm{C}$ and the temperature in the endothelium lies between 60 and $74^{\circ} \mathrm{C}$. An increase in the heat flux phase-lag causes a rise in the corneal temperature whereas an increase in the temperature gradient phase-lag reduces the temperature. There is a need to validate the reliability of the present results by comparing these analytical results with the experimental data. Once the model is validated, it may be used for the better design of laser surgery to treat hyperopia.

\section{Acknowledgement}

The financial assistance for this work received through award no. 09/1230 (0001)/2018-EMR-I from the Council of Scientific and Industrial Research, 
New Delhi is gratefully acknowledged. The author would like to thank the reviewers for their critical review and valuable comments, which improved the paper thoroughly.

\section{References}

[1] Hobiny, A.D., \& Abbas, I.A. (2018). Theoretical analysis of thermal damages in skin tissue in duced by intense moving heat source. International Journal of Heat and Mass Transfer, 124, 1011-1014.

[2] Alzahrani, F.S., \& Abbas, I.A. (2019). Analytical estimations of temperature in a living tissue generated by laser irradiation using experimental data. Journal of Thermal Biology, 85, 10241.

[3] Abbas, I., Hobiny, A., \& Alzahrani, F. (2019). An analytical solution of the bioheat model in a spherical tissue due to laser irradiation. Indian J. Phys., DOI: 10.1007/s12648-019-01581-w.

[4] Hobiny, A., \& Abbas, I. (2019). Thermal response of cylindrical tissue induced by laser irradiation with experimental study. International Journal of Numerical Methods for Heat \& Fluid Flow, 30(8), 4013-4023, DOI: 10.1108/HFF-10-2019-0777.

[5] Ghanmi, A., \& Abbas, I.A. (2019). An analytical study on the fractional transient heating within the skin tissue during the thermal therapy. Journal of Thermal Biology, 82, 229-233.

[6] Hobiny, A., \& Abbas, I. (2019). Analytical solutions of fractional bioheat model in a spherical tissue. An International Journal of Mechanics Based Design of Structures and Machines, DOI: 10.1080/15397734.2019.1702055.

[7] Hobiny, A., Alzahrani, F., Abbas, I., \& Marin, M. (2020). The effect of fractional time derivative of bioheat model in skin tissue induced to laser irradiation. Symmetry, 12, 602, DOI: $10.3390 /$ sym 12040602 .

[8] Hobiny, A.D., \& Abbas, I.A. (2020). Nonlinear analysis of dual-phase lag bio-heat model in living tissues induced by laser irradiation. Journal of Thermal Stresses, 43(4), 503-511.

[9] Brinkmann, R., Koop, N., Droege, G., Grotehusmann, U., Huber, A., Bringruber, R. (1994). Investigations on Laser Therokerato-plasty. Laser Applications in Opthalmology, Proc. SPIE, 2079, 120-130.

[10] Ooi, E.H. \& Ng, E.Y.K. (2009). Ocular temperature distribution: A mathematical perspective. Journal of Mechanics in Medicine and Biology, 9, 199-227.

[11] Mainster, M.A. (1979). Ophthalmic applications of infrared lasers - thermal considerations. Invest. Ophthalmol. Vis. Sci., 18, 414-420.

[12] Rahbar, S., \& Shokooh-Saremi, M.S. (2018). Mathematical modeling of laser linear thermal effects on the anterior layer of the human eye. Optics and Laser Technology, 99, 72-80.

[13] Brinkmann, R., Radt, B., Flamm, C., Kampeier, J., Koop, N., \& Bringruber, R. (2000). Influence of temperature and time on thermally induced forces in corneal collagen and the effect on laser thermo keratoplasty. J. Cataract Refractive Surg., 26(5), 744-754.

[14] Gokul, Kc., Adhikary, P.R., \& Gurung, D.B. (2015). Mathematical Model: Comparative Study of Thermal Effects of Laser in Corneal Refractive Surgeries. Applications and Applied Mathematics, 10(1), 620-633.

[15] Gokul, Kc., Adhikary, P.R., \& Gurung, D.B. (2014). Thermal effects of eyelid in human eye temperature. Journal of Applied Mathematics and Informatics, 32, 649-663.

[16] Manns, F., Borja, D., \& Parel, J.M. (2002). Calculation of corneal temperature and shrinkage during laser thermo keratoplasty (LTK), in: F. Manns, P.G. Söderberg, A. Ho (Eds.). Ophthalmic Technologies XII. Proceedings of the SPIE, 4611, 101-109.

[17] Manns, F., Borja, D., Parel, J.M., \& Smiddy, W. (2003). Semianalytical thermal model for subablative laser heating of homogeneous nonperfused biological tissue: application to laser thermo keratoplasty. J. Biomed. Opt., 8(2), 288-297. 
[18] Ortueta, D.D., Magnago, T., Triefenbach, N., Mosquera, S.A., Sauer, U., \& Brunsmann, U. (2012). In vivo measurement of thermal load during ablation in high speed laser corneal refractive surgery. J. Refract. Surgery, 28, 53-58.

[19] Shibib, K.S. (2013). Finite element analysis of cornea thermal damage due to pulse incidental far IR laser. Lasers Med. Sci., 28, 871-877.

[20] Gheitaghy, A.M., Takabi, B. \& Alizadeh, M. (2014). Modeling of ultrashort pulsed laser irradiation in the cornea based on parabolic and hyperbolic heat equations using electrical analogy. International Journal of Modern Physics C, 25(9), 1450039-1-1450039-17.

[21] Narasimhan, A., \& Sadasivam, S. (2013). Non-Fourier bioheat transfer modelling of thermal damage during retinal laser irradiation. Int. J. Heat and Mass Transf., 60, 591-597.

[22] Pennes, H.H. (1948). Analysis of tissue and arterial blood temperature in the resting forearm. J. Appl. Physiol., 1, 93-122. 